\title{
Gene expression characteristics of growth-inhibited rice seedlings induced by low-energy $\mathbf{N}^{+}$-beam implantation
}

\author{
H. Ya ${ }^{1 *}$, Q. Chen ${ }^{2 *}$, W. Wang ${ }^{2 *}$ and Y. Cheng ${ }^{1}$
}

${ }^{1}$ Life Science Department, Luoyang Normal University, Luoyang, China ${ }^{2}$ Key Laboratory of Ion Beam Bioengineering in Henan Province, Zhengzhou University, Zhengzhou, China

*These authors contributed equally to this study.

Corresponding author: H. Ya

E-mail: 25460678@qq.com

Genet. Mol. Res. 13 (3): 6259-6271 (2014)

Received August 1, 2013

Accepted December 12, 2013

Published August 15, 2014

DOI http://dx.doi.org/10.4238/2014.August.15.9

\begin{abstract}
Using the RNA sequencing, transcriptomes of growthinhibited rice seedlings induced by low-energy $\mathrm{N}^{+}$-beam implantation were analyzed. The results showed that rice genes related to binding, electron carrier activity, transcription factor, auxin signal transduction, photosynthesis, and oxidoreductase activity were involved in the response to ion-beam implantation. Moreover, low-energy $\mathrm{N}^{+}$beam implantation can induce the reactivation of the repressed rice transposable elements. These data are a useful resource for plant ionbeam implantation research.
\end{abstract}

Key words: Transcriptome; Growth-inhibition; RNA-Seq; Low-energy ion-beam implantation 


\section{INTRODUCTION}

Energetic ion radiation can initiate effects in organisms that absorb the energy and undergo physical, physicochemical, and chemical changes. Some changes include molecular insult and produce visible biological defects. Therefore, radiation biology research is essential to predict such deleterious effects. Studies using low-energy ion-beam biotechnology have been implemented to improve crop growth, microbial resistance, and environmental outcomes that have significant economic and societal benefits. Although low-energy ion-beam implantation has obvious benefits, such energy can induce mutagenesis, injury, and plant death (Tanaka et al., 2010). Low-energy ion-beam irradiation can cause nuclear and chromosomal defects, resulting in DNA deletions, duplications, and transposition of DNA fragments (Shikazono et al., 2001; Feng et al., 2007; Ya et al., 2010). Other plant responses to ion-beam irradiation include oxidative stress, free radical generation, and changes in signal transduction, such as long-distance transfer of radiation effect signals and alterations in the abscisic acid signal pathway (Feng et al., 2006; Chen et al., 2010). Presently, insufficient research exists to fully describe mechanisms by which low-energy ion-beam implantation affects plants, especially mechanisms by which low-energy implantation can give rise to desired plant mutations without chromosomal damage (Yamaguchi et al., 2010). Thus, research is needed to improve ionbeam implantation in plants to improve bioengineering methods for agricultural use.

Recent developments in transcriptomics, bioinformatics, and high-throughput sequencing technologies (RNA-Seq) have elucidated processes at the transcriptional level. Systems biology has also undergone a shift from traditional molecular biology, which merely concerns itself with individual genes and proteins. Systems biology allows scientists to explore the whole biological systems with respect to function (Benner, 2008). In systemic biological studies, however, the effects of low-energy ion-beam implantation on biological systems are unclear. To investigate such effects, we used microarrays to study the preliminary characteristics of transcriptome-related growth stimulation effects of ion-beam irradiation in rice (Ya et al., 2012). Such microarray studies require plant sample sequence information to fully elucidate the identity of new transcripts in plant processes that are responsive to low-energy implantation. In addition, microarray limitations include sensitivity issues of the hybridization technology. Detecting targets of low abundance or repeated sequences, such as fusion gene transcription and polycistronic transcription, remains complicated. Such information is needed to study the importance of new transcripts and low abundance and repeated sequences in plants irradiated with low-energy ion beams. RNA-Seq technology is advantageous because transcriptome expression profiles of arbitrary species can be studied without advance sequence information. Thus, this technology can be used to determine gene structure and gene splicing patterns, as well as to detect rare or new transcripts, which is suitable for our studies of complex transcriptome contributions to specific biological processes (Wang et al., 2009). High-energy irradiation is well studied in animals, but low-energy irradiation studies are not well represented in the scientific literature. Thus, our transcriptomic research based on RNASeq technology is innovative and will provide new opportunities and biological perspectives for the use of ion-beam implantation in plants.

Previously, research on ion-beam implantation focused on the immediate cause of the mutagenesis (Shikazono et al., 2001; Maekawa et al., 2003; Yamaguchi et al., 2003). The molecular mecanism of generational effects of ion-beam irradiation is seldom investigated 
because first-generation biological effects are rarely inherited by subsequent generations. Studying these molecular mechanisms of ion-beam implantation effects can improve studies of mutagenesis mechanisms, specifically those that affect plant growth inhibition (injury) induced by low-energy ion-beam implantation. Most plant mutations induced by low-energy ion-beam implantation are derived from first-generation injuries. Using RNA-Seq technology to explore gene expression profiles related to plant growth inhibition induced by low-energy ion-beam irradiation, we can speculate about plant cellular events based on the metabolism or biological processes that are affected by differential gene expression.

\section{MATERIAL AND METHODS}

\section{Plant material}

We cultivated Xindao-18 (Oryza sativa L. subsp. japonica Kato) rice seeds to mature seedlings and collected the panicle. We removed the glume manually to avoid injury to the rice embryo. Then, complete and similarly-sized seeds were selected as experimental material.

\section{Low-energy ion-beam implantation}

To irradiate seeds with a low-energy ion beam, rice seeds (moisture content: 6.7\%) were vertically fixed on a cork with the embryo facing upward. The cork was then placed on a culture dish $(10 \times 10 \mathrm{~cm})$. Samples were divided into six groups, and five of them were implanted by an $\mathrm{N}^{+}$beam with a current intensity of $2 \mathrm{~mA}$, energy of $40 \mathrm{Kev}$, and ion fluences of $1 \times 10^{17}, 2 \times 10^{17}, 4 \times 10^{17}, 6 \times 10^{17}$, and $8 \times 10^{17} \mathrm{~N}^{+} / \mathrm{cm}^{2}$, respectively. Ion implantation was conducted in a vacuum $\left(10^{-2} \mathrm{MPa}\right)$ target chamber [implanter model: Ion Beam Bioengineering Facility (UIL.0.512, TNV, Russia]. A seed group that was not implanted with low-energy served as a control. Each group was replicated three times, and each replication contained 150 rice seeds.

\section{Biomaterial cultivation and germination rate, seedling height, and root length measurements}

An aseptic filter paper was placed on the bottom of an aseptic culture dish moistened with aseptic water. Implanted seeds and control seeds were placed on the filter paper, and the culture dish was placed in a climate box (12 h light/12 h dark cycles) with a constant temperature $\left(28^{\circ} \mathrm{C}\right)$. Every $12 \mathrm{~h}, 10 \mathrm{~mL}$ aseptic water was uniformly dripped on the filter paper in the culture dish to maintain moisture. After culturing for $96 \mathrm{~h}$, we randomly collected 100 seed buds [including bud $(\sim 3 \mathrm{~cm})$ and root] from each sample group. Buds $(\mathrm{N}=100)$ were stored at $-80^{\circ} \mathrm{C}$ and later sequenced and subjected to total RNA extraction of mixed strains. Remaining buds were kept in culture to the seventh day $(168 \mathrm{~h})$. The seed germination rate (germination number/total seeds), seedling height (length from hypocotyl to top of longest leaf), and root length (length of longest root: length from hypocotyl to root tip) of these buds were measured. 


\section{Preparation of sequencing samples}

Because a seven-day germination should provide ample time for early gene expression, rice buds cultured for $96 \mathrm{~h}$ were selected as material for RNA extraction and RNA-seq. Fifty of the above 100 buds cultured for $96 \mathrm{~h}$ and similar in height were selected from each treated material group for the total RNA pool extraction. In experiments, we had one control RNA pool (Sample 1, the first sequencing sample) and five RNA pools from the five groups that were treated with ion fluences of $1 \times 10^{17}, 2 \times 10^{17}, 4 \times 10^{17}, 6 \times 10^{17}$, and $8 \times 10^{17} \mathrm{~N}^{+} /$ $\mathrm{cm}^{2}$, respectively. Based on biological effects measured, equal $10 \mathrm{~g}$ RNA of two RNA pools from two ion-beam implanted groups with ion fluences of $1 \times 10^{17}$ and $2 \times 10^{17} \mathrm{~N}^{+} / \mathrm{cm}^{2}$ were mixed to form the second sequencing sample (Sample 2: no significant growth inhibition differences compared to control). Next, equal $10 \mu \mathrm{g}$ RNA of two RNA pools from two ion-beam implanted groups with ion fluences of $6 \times 10^{17}$ and $8 \times 10^{17} \mathrm{~N}^{+} / \mathrm{cm}^{2}$ were combined to form the third sequencing sample (Sample 3: sample with significant growth inhibition differences compared to control).

\section{RNA extraction, cDNA library preparation, and sequencing}

Total RNA was extracted from whole seedlings using Trizol reagent (Invitrogen, USA) and purified with the RNeasy Plant Mini Kit (Qiagen, USA). The RNA quality was verified on a Bioanalyzer 2100 (Aligent, USA); the RNA integrity number values were $>8.5$ for all samples. Sequencing libraries were prepared according to manufacturer instructions (Illumina, USA). Poly-A-containing mRNA was isolated from the total RNA, subjected to two purification rounds using poly-T oligo-attached magnetic beads, and fragmented using an RNA fragmentation kit. First-strand cDNA was generated using reverse transcriptase and random primers. After second-strand cDNA synthesis and adaptor ligation, 200-bp cDNA fragments were isolated using gel electrophoresis and amplified with 18 cycles of polymerase chain reaction. Products were loaded onto an Illumina HiSeq2000 instrument and subjected to 100 cycles of paired-end ( 2 x $100 \mathrm{bp})$ sequencing. The processing of fluorescent images into sequences, base calling, and quality value calculations were performed using the Illumina data processing pipeline (version 1.8). The sequence reads were submitted to the GenBank gene expression omnibus database under accession number GSE45908 (http://www.ncbi.nlm.nih. gov/geo/query/acc.cgi?acc=GSE45908).

\section{Mapping of short reads and assessment of differential gene expression}

Raw reads were filtered to obtain high-quality reads by removing low-quality reads containing more than $50 \%$ bases with $\mathrm{Q}<30$. After trimming low-quality bases $(\mathrm{Q}<30)$ from the $5^{\prime}$ and $3^{\prime}$ ends of the remaining reads, the resulting high-quality reads were mapped onto the Nipponbare reference genome (MSU v7.0) using Tophat v2.0.5. Differential expression was estimated and tested with the edgeR software package ( $\mathrm{R}$ version: 2.14, edgeR version: 2.3.52) (Robinson et al., 2010). Gene expression was quantified in terms of reads per kilobase per million mapped reads [RPKM = total exon reads/mapped reads (millions) $\mathrm{x}$ exon length $(\mathrm{Kb})]$ (Mortazavi et al., 2008). The false-discovery rate (FDR) was calculated and the fold change (FC) and $\log 2$ values of FC were estimated. Transcripts with an FDR $\leq 0.05$ and an 
estimated absolute $\log 2$ (FC) $\geq 1$ were considered to be significantly differentially expressed.

\section{RESULTS}

\section{Descriptive statistics of seed germination rate, seedling height, and root length}

Data revealed no statistically significant differences in the seed germination rate between implanted samples and control samples despite the seed germination rates of all implanted samples being greater than that of the control group.

In comparisons of seedling root lengths (Figure 1A), seedling heights of samples treated with ion fluences of $6 \times 10^{17}$ and $8 \times 10^{17} \mathrm{~N}^{+} / \mathrm{cm}^{2}$ were significantly different than those of control samples, but no statistically significant differences in seedling heights were observed with ion fluence treatments of $1 \times 10^{17}, 2 \times 10^{17}$, and $4 \times 10^{17} \mathrm{~N}^{+} / \mathrm{cm}^{2}$ compared to the control samples. Thus, seedling root growth may be inhibited by ion fluences of $6 \times 10^{17}$ and $8 \times 10^{17} \mathrm{~N}^{+} / \mathrm{cm}^{2}$.

In comparisons of seedling heights (Figure 1B), a statistically significant difference was observed in samples treated with ion fluences of $4 \times 10^{17}, 6 \times 10^{17}$, and $8 \times 10^{17} \mathrm{~N}^{+} / \mathrm{cm}^{2}$ compared to control samples. No statistically significant differences in seedling heights of samples treated with ion fluences of $1 \times 10^{17}$ and $2 \times 10^{17} \mathrm{~N}^{+} / \mathrm{cm}^{2}$ were observed compared to control samples. Therefore, seedling height appears to be inhibited by ion fluences of $4 \times 10^{17}$, $6 \times 10^{17}$, and $8 \times 10^{17} \mathrm{~N}^{+} / \mathrm{cm}^{2}$.

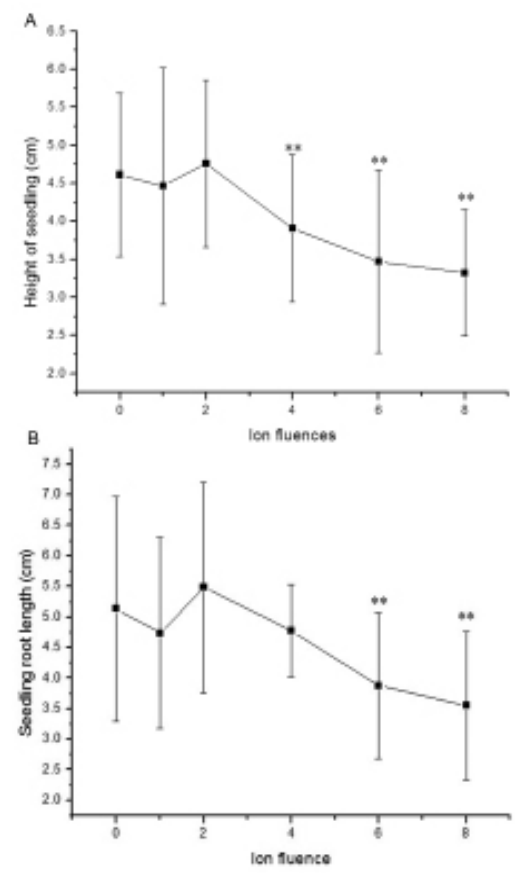

Figure 1. Comparisons of seedling height and root length among different groups. A. Seedling root length. B. Seedling height. $0,1,2,4,6$, and 8 refer to the following treatments: blank control, ion fluence of $1 \times 10^{17}, 2 \times 10^{17}$, $4 \times 10^{17}, 6 \times 10^{17}$, and $8 \times 10^{17} \mathrm{~N}^{+} / \mathrm{cm}^{2}$, respectively. ${ }^{* *}$ Significant difference with $\mathrm{P}<0.01$. 
Our data led us to hypothesize that the stronger ion fluences of $6 \times 10^{17}$ and $8 \times 10^{17}$ $\mathrm{N}^{+} / \mathrm{cm}^{2}$ could induce biological damage, but ion fluences of $1 \times 10^{17}$ and $2 \times 10^{17} \mathrm{~N}^{+} / \mathrm{cm}^{2}$ did not injure seedlings. According to this result, we prepared three sequencing samples: Sample 1 (control), Sample 2 (mixed RNAs from the samples that were implanted by ion fluences of 1 $\mathrm{x} 10^{17}$ and $2 \times 10^{17} \mathrm{~N}^{+} / \mathrm{cm}^{2}$ ), and Sample 3 (mixed RNAs from the samples that were implanted by ion fluences of $6 \times 10^{17}$ and $\left.8 \times 10^{17} \mathrm{~N}^{+} / \mathrm{cm}^{2}\right)$.

\section{Mapping reads to the rice genome}

Paired-end sequencing was performed on the three samples using the Illumina HiSeq2000 platform, generating 12,792,715 (Sample 1), 12,170,872 (Sample 2), and 19,177,117 (Sample 3) 2 x 100-bp reads. Raw sequence data are available in the National Center for Biotechnology Information Sequence Read Archive (GSE45908) for further analysis. In total, 8.92 gigabase data were generated from the three samples, and high-quality 100-bp reads including 8.05 gigabase were selected. We pooled the short reads and aligned them against the Nipponbare reference genome (MSU V7) using Tophat v2.0.5. Of the reads, $64.4-72.4 \%$ were mapped to exonic regions, $19.1-20.6 \%$ were mapped to untranslated regions, $2.36-2.49 \%$ were mapped to intronic regions, and $4.95-14.0 \%$ were mapped to intergenic regions.

\section{Identification of differentially expressed genes (DEGs)}

DEGs were determined by comparing the expressed genes in Sample 2 with those in Sample 1 (control) and also from comparing the expressed genes in Sample 3 with those in Sample 1. Gene expression was measured as RPKM. Putative DEGs were identified using the following criteria: (1) FDR $\leq 0.05$ and (2) FC $\geq 2$. With these criteria, the numbers of DEGs identified are described in Table 1.

Table 1. Differentially expressed genes (DEGs).
\begin{tabular}{lccc} 
& \\
\hline Comparisons & DEGs (N) & Upregulated transcripts & Downregulated transcripts \\
\hline Sample 2 vs Sample 1 & 554 & 262 & 282 \\
Sample 3 vs Sample 1 & 776 & 283 & 493 \\
Sample 3 vs Sample 2 & 726 & 352 & 374 \\
\hline
\end{tabular}

One hundred seven DEGs overlapped in the Sample 2 vs Sample 1 and Sample 3 vs Sample 1 comparisons, which included 58 downregulated DEGs, 48 upregulated DEGs, and 8 DEGs that presented other regulation models.

\section{Functional classification by Gene Ontology (GO)}

Agrigo was used for the functional classification of DEGs, and 307 of the 554 DEGs in Sample 2 vs Sample 1 were classified into 83 GO molecular function terms, 21 GO cellular component terms, and $120 \mathrm{GO}$ biological process category terms (Du et al., 2010). Only six terms in the molecular function categories were significant (FDR $<0.05$, Table 2). In the molecular function category, binding was prominently represented. For Sample 3 vs Sample 1, 453 of 773 DEGs were classified into $96 \mathrm{GO}$ molecular function terms, 28 cellular component terms, and 130 biological process category terms. Of these, 14 terms in molecular function categories, 14 terms in biological process categories, and two terms in cellular component categories were statistically significant (Table 3 ). In the molecular function category, binding, 
electron carrier activity, and oxidoreductase activity were well represented, whereas "extracellular region" dominated the cellular component category. In the biological process category, photosynthesis, response, and energy were prominently represented.

From comparisons of Sample 3 vs Sample 2, 396 of 726 DEGs were classified into 89 GO molecular function terms, 28 cellular component terms, and 111 biological process category terms, and two molecular function categories and six biological process categories were statistically significant. No cellular component categories were significant (Table 4). In the molecular function category, hydrolase activity was prominently represented, and "photosynthesis" dominated the biological process category.

Table 2. Significant Gene Ontology (GO) of differentially expressed genes (DEG) terms in Sample $2 v s$
Sample 1.
\begin{tabular}{lclccr} 
\\
\hline GO term & Ontology & Description & No. of DEGs & P value & FDR \\
\hline GO:0000166 & F & Nucleotide binding & 74 & 0.00063 & 0.043 \\
GO:0017076 & F & Purine nucleotide binding & 68 & 0.0005 & 0.043 \\
GO:0030145 & F & Manganese ion binding & 6 & 0.00013 & 0.043 \\
GO:0045735 & F & Nutrient reservoir activity & 6 & 0.00046 & 0.043 \\
GO:0032555 & F & Purine ribonucleotide binding & 65 & 0.00061 & 0.043 \\
GO:0042802 & F & Identical protein binding & 5 & 0.00079 & 0.043 \\
\hline
\end{tabular}

$\mathrm{F}=$ molecular function category; FDR $=$ false-discovery rate.

Table 3. Significant Gene Ontology (GO) of differentially expressed genes (DEG) terms in Sample $3 v s$ Sample 1.

\begin{tabular}{|c|c|c|c|c|c|}
\hline GO term & Ontology & Description & No. of DEGs & P value & FDR \\
\hline GO:0009765 & $\mathrm{P}$ & Photosynthesis, light harvesting & 11 & $2.90 \mathrm{E}-15$ & $2.00 \mathrm{E}-12$ \\
\hline GO:0019684 & $\mathrm{P}$ & Photosynthesis, light reaction & 13 & $1.50 \mathrm{E}-12$ & $5.40 \mathrm{E}-10$ \\
\hline GO:0015979 & $\mathrm{P}$ & Photosynthesis & 16 & $7.50 \mathrm{E}-11$ & $1.80 \mathrm{E}-08$ \\
\hline GO:0006979 & $\mathrm{P}$ & Response to oxidative stress & 18 & $4.00 \mathrm{E}-08$ & $7.00 \mathrm{E}-06$ \\
\hline GO:0006091 & $\mathrm{P}$ & Generation of precursor metabolites and energy & 21 & $6.00 \mathrm{E}-07$ & $8.50 \mathrm{E}-05$ \\
\hline GO:0042221 & $\mathrm{P}$ & Response to chemical stimulus & 20 & $2.30 \mathrm{E}-06$ & 0.00027 \\
\hline GO:0010876 & $\mathrm{P}$ & Lipid localization & 11 & $4.50 \mathrm{E}-06$ & 0.00035 \\
\hline GO:0006950 & $\mathrm{P}$ & Response to stress & 38 & 4.40E-06 & 0.00035 \\
\hline GO:0006869 & $\mathrm{P}$ & Lipid transport & 11 & $4.50 \mathrm{E}-06$ & 0.00035 \\
\hline GO:0050896 & $\mathrm{P}$ & Response to stimulus & 40 & $2.20 \mathrm{E}-05$ & 0.0016 \\
\hline GO:0005975 & $\mathrm{P}$ & Carbohydrate metabolic process & 35 & $3.30 \mathrm{E}-05$ & 0.0021 \\
\hline GO:0071669 & $\mathrm{P}$ & Plant-type cell wall organization or biogenesis & 5 & 0.00034 & 0.019 \\
\hline GO:0009664 & $\mathrm{P}$ & Plant-type cell wall organization & 5 & 0.00034 & 0.019 \\
\hline GO:0055114 & $\mathrm{P}$ & Oxidation reduction & 14 & 0.00048 & 0.024 \\
\hline GO:0016684 & $\mathrm{F}$ & Oxidoreductase activity, acting on peroxide as acceptor & 18 & $6.80 \mathrm{E}-08$ & $1.10 \mathrm{E}-05$ \\
\hline GO:0009055 & $\mathrm{F}$ & Electron carrier activity & 44 & $5.40 \mathrm{E}-08$ & $1.10 \mathrm{E}-05$ \\
\hline GO:0004601 & $\mathrm{F}$ & Peroxidase activity & 18 & $6.80 \mathrm{E}-08$ & $1.10 \mathrm{E}-05$ \\
\hline GO:0016209 & $\mathrm{F}$ & Antioxidant activity & 18 & $2.50 \mathrm{E}-07$ & $3.10 \mathrm{E}-05$ \\
\hline GO:0004553 & $\mathrm{F}$ & Hydrolase activity, hydrolyzing O-glycosyl compounds & 26 & $9.00 \mathrm{E}-07$ & $9.00 \mathrm{E}-05$ \\
\hline GO:0016798 & $\mathrm{F}$ & Hydrolase activity, acting on glycosyl bonds & 27 & $1.90 \mathrm{E}-06$ & 0.00016 \\
\hline GO:0020037 & $\mathrm{F}$ & Heme binding & 28 & $3.10 \mathrm{E}-05$ & 0.0022 \\
\hline GO:0005506 & $\mathrm{F}$ & Iron ion binding & 31 & $4.00 \mathrm{E}-05$ & 0.0022 \\
\hline GO:0046906 & $\mathrm{F}$ & Tetrapyrrole binding & 28 & $3.80 \mathrm{E}-05$ & 0.0022 \\
\hline GO:0016491 & $\mathrm{F}$ & Oxidoreductase activity & 63 & 0.00011 & 0.0054 \\
\hline GO:0048037 & $\mathrm{F}$ & Cofactor binding & 23 & 0.00037 & 0.017 \\
\hline GO:0008171 & $\mathrm{F}$ & O-methyltransferase activity & 6 & 0.0005 & 0.021 \\
\hline GO:0016829 & $\mathrm{F}$ & Lyase activity & 14 & 0.0012 & 0.045 \\
\hline GO:0005507 & $\mathrm{F}$ & Copper ion binding & 9 & 0.0013 & 0.046 \\
\hline GO:0005576 & $\mathrm{C}$ & Extracellular region & 20 & $2.80 \mathrm{E}-07$ & $3.10 \mathrm{E}-05$ \\
\hline GO:0048046 & $\mathrm{C}$ & Apoplast & 10 & $1.10 \mathrm{E}-05$ & 0.00062 \\
\hline
\end{tabular}

$\mathrm{F}=$ molecular function category; $\mathrm{C}=$ cellular component; $\mathrm{P}=$ biological process category; 


\begin{tabular}{|c|c|c|c|c|c|}
\hline GO term & Ontology & Description & No. in DEGs & $\mathrm{P}$ value & FDR \\
\hline GO:0009765 & $\mathrm{P}$ & Photosynthesis, light harvesting & 11 & $6.70 \mathrm{E}-16$ & $4.00 \mathrm{E}-13$ \\
\hline GO:0015979 & $\mathrm{P}$ & Photosynthesis & 19 & $5.30 \mathrm{E}-15$ & $1.60 \mathrm{E}-12$ \\
\hline GO:0019684 & $\mathrm{P}$ & Photosynthesis, light reaction & 12 & $6.80 \mathrm{E}-12$ & $1.30 \mathrm{E}-09$ \\
\hline GO:0006091 & $\mathrm{P}$ & Generation of precursor metabolites and energy & 17 & $1.90 \mathrm{E}-05$ & 0.0027 \\
\hline GO:0046148 & $\mathrm{P}$ & Pigment biosynthetic process & 5 & $5.70 \mathrm{E}-05$ & 0.0067 \\
\hline GO:0042440 & $\mathrm{P}$ & Pigment metabolic process & 5 & 0.00012 & 0.011 \\
\hline GO:0004553 & $\mathrm{F}$ & Hydrolase activity, hydrolyzing O-glycosyl compounds & 20 & $8.90 \mathrm{E}-05$ & 0.02 \\
\hline GO:0043566 & $\mathrm{F}$ & Structure-specific DNA binding & 5 & $6.90 \mathrm{E}-05$ & 0.02 \\
\hline
\end{tabular}

$\mathrm{F}=$ molecular function category; $\mathrm{P}=$ biological process category.

\section{Kyoto Encyclopedia of Gene and Genome (KEGG) pathway analysis}

To identify metabolic pathways that are responsive to ion-beam implantation adaptation, pathway-based analysis was performed using the KEGG pathway database. No significant pathways of DEGs were observed in Sample 3 vs Sample 1 or Sample 3 vs Sample 2 comparisons except the photosynthesis-antenna protein pathway (Pathwayid: map00196) in Sample 3. No significantly enriched pathways of DEG were found to be involved Samples 2 and 3 were compared.

Of the top 5 most-represented pathways among the samples (not shown), biosynthesis of secondary metabolites was the overlapping prominently enriched pathway. These data suggest that secondary metabolites, as a common pathway, were involved in adaptive regulation to low-energy $\mathrm{N}^{+}$-beam implantation in rice.

\section{DISCUSSION}

Our analysis of gene expression profiles for growth-inhibited biological effects arising from ion-beam implantation in rice seedlings revealed transcriptome characteristics during seed germination. These data suggest that DEGs clustered into groups that were associated with unique requirements of ion-implantation adaptation. These data provide information about many candidate genes that can be more intensely studied to learn the molecular mechanisms of ion-beam implantation plant injury and its use in crop improvement.

\section{Downregulated photosynthesis contributes to the damaged biological effects aris- ing from ion-beam implantation}

Light-harvesting chlorophyll a/b-binding protein (LHCP) is one of the most abundant chloroplast proteins found in plants. LHCP's chief function is to collect and transfer light energy to photosynthetic reaction centers (Xia et al., 2012). Many homologous genes encoding LHCPs from various plant species belong to one of the 10 gene family members (Teramoto et al., 2001). Under changing light conditions, the reversible phosphorylation of LHCP a/bbinding proteins (LHCII) represents a system for balancing excitation energy between photosystems I and II (Liu and Shen, 2004). The 11 downregulated DEGs (chlorophyll a/b-binding protein) involved in the photosynthesis pathway were less expressed in Samples 2 and 3 than in the control sample (Table 5). Such diminished expression reduces light harvesting and syn- 
thesis of plant energy. In agreement with this concept, the seedling root length and height in Sample 3 were diminished, suggesting that the growth of samples treated with ion fluences of $6 \times 10^{17}$ and $8 \times 10^{17} \mathrm{~N}^{+} / \mathrm{cm}^{2}$ was inhibited through the downregulated expression of genes related to chlorophyll a/b-binding protein.

Table 5. Downregulated differentially expressed genes (DEGs) involved in the photosynthesis pathway.
\begin{tabular}{lcc}
\hline Gene_ID & Log2 (FC) Sample 3 vs Sample 1 & Log2 (FC) Sample 3 vs Sample 2 \\
\hline LOC_Os01g41710 & -5.12 & -5.31987 \\
LOC_Os02g10390 & -4.46 & -5.13882 \\
LOC_Os03g39610 & -6.25 & -6.60422 \\
LOC_Os04g38410 & -2.13 & -2.7851 \\
LOC_Os06g21590 & -2.09 & -2.73821 \\
LOC_Os07g37240 & -1.52 & -1.91876 \\
LOC_Os07g37550 & -2.85 & -3.25119 \\
LOC_Os07g38960 & -1.63 & -2.12569 \\
LOC_Os08g33820 & -4.38 & -4.88249 \\
LOC_Os09g17740 & -5.94 & -6.27338 \\
\hline
\end{tabular}

$\mathrm{FC}=$ fold change.

\section{Transcription factors (TFs) involved in the biological effects induced by ion-beam implantation}

TFs are essential for the regulation of gene expression and are, as a consequence, found in all living organisms (Chen and Rajewsky, 2007). Differentially expressed TFs were explored using the database of rice TFs (http://drtf.cbi.pku.edu.cn/) and the rice annotation project database (http://rapdb.dna.affrc.go.jp). In Sample 2, we identified 19 TFs differentially expressed compared to the control sample (Table 5), including 52.63\% (10/19) upregulated TFs and $47.37 \%(9 / 19)$ downregulated TFs. In contrast, of the 20 differentially expressed TFs identified in Sample 3 compared to control (Table 6), only 25\% (5/20) were upregulated; $75 \%$ $(15 / 20)$ were downregulated. Most of the downregulated TFs were related to rice growth inhibition induced by ion-beam implantation.

The differentially expressed TFs $(\mathrm{N}=19)$ in Sample 2 were classified into 9 TF families and one non-classified family (Table 6). Meanwhile, the 20 differentially expressed TFs in Sample 3 were classified into $10 \mathrm{TF}$ families and one non-classified family (Table 7). The basic helix-loop-helix (bHLH) and GARP-G2-like TF families were overlapping and differentially expressed TF families in Samples 2 and 3. The bHLH TFs comprise the largest family of plant TFs, and they are important for eukaryotic developmental processes (Wang et al., 2008). Thus, differentially expressed bHLH family TFs warrant further study to ascertain their roles in plant adaptation to low-energy ion-beam implantation.

Hormones are well-characterized plant signaling molecules that mediate physiological responses. For example, auxins promote root initiation and induce pre-existing root growth and adventitious (branching) root formation. In low concentrations, auxin can inhibit ethylene formation and the transport of plant precursors; however, high auxin concentrations can induce ethylene synthesis (Nemhauser et al., 2000). Our data show that TF expression related to AUX/ IAA (auxin/indole acetic acid) decreased in Sample 3 with growth inhibition. In addition, we observed a downregulated DEG that was annotated as the auxin-induced protein 5NG4 [LOC Os08g44750, $\log 2(\mathrm{FC})=-1.7]$. These findings suggest that low-energy $\mathrm{N}^{+}$-beam implantation 
damaged the regulation of TFs that were related to AUX/IAA, subsequently downregulating them and inhibiting growth. Such data suggest that auxin may be critical in adaptive processes in rice seedlings after nitrogen-beam implantation. In addition, ion-beam implantation may alter seedling development during germination via plant hormone regulation.

\begin{tabular}{|c|c|c|c|c|}
\hline Gene_ID & $\log 2(\mathrm{FC})$ & P value & Q value & TF family \\
\hline LOC_Os03g14720 & $-1.80 \mathrm{e}+308$ & $6.98 \mathrm{E}-07$ & 0.0002723 & unclassified \\
\hline $\mathrm{LOC}^{-} \mathrm{Os} 04 \mathrm{~g} 53460$ & $-1.80 e+308$ & $8.01 \mathrm{E}-07$ & 0.0003076 & unclassified \\
\hline $\mathrm{LOC}^{-} \mathrm{Os} 07 \mathrm{~g} 42400$ & $1.80 \mathrm{e}+308$ & 0.0001633 & 0.0167781 & unclassified \\
\hline LOC_Os12g39070 & $-1.80 \mathrm{e}+308$ & 0.0002012 & 0.0191328 & unclassified \\
\hline $\mathrm{LOC}^{-} \mathrm{Os} 12 \mathrm{~g} 29520$ & $1.80 \mathrm{e}+308$ & $1.59 \mathrm{E}-09$ & $1.87 \mathrm{E}-06$ & $\mathrm{ARF}$ \\
\hline LOC_Os01g38610 & 1.38 & 0.0006711 & 0.042414 & bHLH \\
\hline $\mathrm{LOC}^{-} \mathrm{Os} 02 \mathrm{~g} 56140$ & $-1.80 e+308$ & 0.0007342 & 0.0445114 & bHLH \\
\hline $\mathrm{LOC}_{-}^{-} \mathrm{Os} 12 \mathrm{~g} 41650$ & $1.80 \mathrm{e}+308$ & 0.0007107 & 0.0437781 & bHLH \\
\hline LOC_Os07g49460 & $1.80 \mathrm{e}+308$ & $5.69 \mathrm{E}-05$ & 0.0079732 & $\mathrm{C} 2 \mathrm{C} 2$-CO-like \\
\hline $\mathrm{LOC}^{-} \mathrm{Os} 03 \mathrm{~g} 48970$ & 3.84 & 0.0008569 & 0.0488504 & CCAAT-HAP2 \\
\hline $\mathrm{LOC}^{-} \mathrm{Os} 06 \mathrm{~g} 35140$ & $-1.80 e+308$ & 8.17E-07 & 0.0003127 & GARP-G2-like \\
\hline $\mathrm{LOC}^{-} \mathrm{Os} 07 \mathrm{~g} 48596$ & $1.80 \mathrm{e}+308$ & 0.0004637 & 0.0330234 & GARP-G2-like \\
\hline LOC_Os12g13570 & $-1.80 e+308$ & $5.64 \mathrm{E}-05$ & 0.007933 & MYB \\
\hline LOC_Os01g09550 & $1.80 \mathrm{e}+308$ & $8.24 \mathrm{E}-05$ & 0.010249 & NAC \\
\hline $\mathrm{LOC}^{-} \mathrm{Os} 05 \mathrm{~g} 10620$ & $-1.80 e+308$ & $8.20 \mathrm{E}-05$ & 0.0102062 & NAC \\
\hline $\mathrm{LOC}^{-} \mathrm{Os} 06 \mathrm{~g} 36480$ & $1.80 \mathrm{e}+308$ & $1.96 \mathrm{E}-05$ & 0.0036768 & NAC \\
\hline $\mathrm{LOC}^{-} \mathrm{Os} 01 \mathrm{~g} 14370$ & $-1.80 \mathrm{e}+308$ & 4.02E-06 & 0.0010822 & TAZ \\
\hline $\mathrm{LOC}^{-} \mathrm{Os} 01 \mathrm{~g} 66890$ & $1.80 \mathrm{e}+308$ & 0.0001319 & 0.0145273 & TAZ \\
\hline LOC_Os12g02420 & $-1.80 \mathrm{e}+308$ & 7.61E-05 & 0.0096016 & WRKY \\
\hline
\end{tabular}

Table 7. Differentially expressed transcription factors (TFs): Sample 3 vs Sample 1.

\begin{tabular}{lcccc}
\hline Gene_ID & Log2 $(\mathrm{FC})$ & P value & Q value & TF family \\
\hline LOC_Os06g10780 & -1.82 & 0.0007632 & 0.0456146 & AP2/EREBP \\
LOC_Os08g36920 & -1.84 & 0.0008048 & 0.0469286 & AP2/EREBP \\
LOC_Os11g32110 & $-1.80 \mathrm{e}+308$ & $1.19 \mathrm{E}-05$ & 0.0025616 & ARF \\
LOC_Os03g43400 & -1.87 & $7.21 \mathrm{E}-05$ & 0.0093002 & AUX/IAA \\
LOC_Os03g43410 & -1.44 & $3.16 \mathrm{E}-05$ & 0.0053021 & AUX/IAA \\
LOC_Os12g40900 & -1.20 & 0.0004779 & 0.0336361 & AUX/IAA \\
LOC_Os05g27090 & -1.52 & 0.0007388 & 0.0446672 & bHLH \\
LOC_Os06g08500 & -2.20 & $3.81 \mathrm{E}-05$ & 0.0059848 & bHLH \\
LOC_Os12g43620 & -1.30 & 0.0007675 & 0.0457074 & bHLH \\
LOC_Os07g13260 & $1.80 \mathrm{e}+308$ & $9.75 \mathrm{E}-05$ & 0.0115941 & C2C2-Dof \\
LOC_Os07g48570 & -2.78 & $1.06 \mathrm{E}-09$ & $0.39 \mathrm{E}-06$ & C2C2-Dof \\
LOC_Os06g13670 & -1.65 & 0.0008647 & $1.16 \mathrm{E}-08$ & E2F/DP \\
LOC_Os01g74020 & 2.75 & $5.14 \mathrm{E}-12$ & GARP-G2-like \\
LOC_Os06g24070 & -1.25 & 0.0004854 & 0.0340409 & GARP-G2-like \\
LOC_Os03g51970 & $-1.80 \mathrm{e}+308$ & $4.39 \mathrm{E}-05$ & GRF \\
LOC_Os07g03770 & -1.79 & 0.0008369 & 0.0480702 & HB \\
LOC_Os02g02370 & -1.69 & 0.0006658 & 0.0421923 & MYB \\
LOC_Os03g63150 & 2.39 & 0.0003292 & 0.0263175 & unclassified \\
LOC_Os04g23890 & 1.43 & $4.50 \mathrm{E}-05$ & 0.0066587 & unclassified \\
LOC_Os05g51150 & 1.99 & 0.0001418 & 0.0151958 & unclassified \\
\hline
\end{tabular}

\section{Signaling}

Cell signaling modulates the biological effects of low-energy ion implantation (Chen et al., 2010). Of the eight DEGs classified as biological signaling processes in Sample 2 compared to the control sample, six were downregulated, and two were upregulated (Table 8). Of the DEGs from Sample $3(\mathrm{~N}=11)$ compared to the control sample classified into biological 
signaling processes, six were downregulated, whereas five were upregulated (Table 9). Signaling proteins related to kinases and $\mathrm{Ca}^{2+} /$ calmodulin-dependent protein kinase (CAMK) are involved in low-energy implantation adaptation. Kinases, including CAMK, transmit signals and control complex cellular processes (Manning et al., 2002; Scheeff and Bourne, 2005). Their diversity and roles in signaling suggest that they are worthy of investigation, especially with respect to plant adaption to low-energy ion-beam implantation.

Table 8. Differentially expressed genes (DEGs) related to signaling: Sample 2 vs Sample 1.

\begin{tabular}{lrrll}
\hline Gene_ID & \multicolumn{1}{c}{$\log 2(\mathrm{FC})$} & \multicolumn{1}{c}{ P value } & Q value & Description \\
\hline LOC_Os07g49460 & $-1.80 \mathrm{e}+308$ & $9.61 \mathrm{E}-05$ & 0.0115213 & Response regulator receiver domain-containing protein \\
LOC_Os03g59590 & $1.80 \mathrm{e}+308$ & 0.0002303 & 0.0208661 & ATP/GTP/Ca ${ }^{2+}$-binding protein \\
LOC_Os02g39560 & $-1.80 \mathrm{e}+308$ & $7.07 \mathrm{E}-06$ & 0.0016997 & Non-receptor tyrosine kinase spore lysis A \\
LOC_Os02g06570 & $-1.80 \mathrm{e}+308$ & $2.87 \mathrm{E}-05$ & 0.0049155 & CAMK_KIN1/SNF1/Nim1_like.13-CAMK \\
LOC_Os03g20720 & $-1.80 \mathrm{e}+308$ & 0.0001048 & 0.0122201 & GTPase-activating protein, putative \\
LOC_Os10g37410 & $-1.80 \mathrm{e}+308$ & 0.0001033 & 0.0121173 & TBC domain-containing protein \\
LOC_Os02g02840 & $1.80 \mathrm{e}+308$ & 0.0008355 & 0.0480121 & Ras-related protein \\
LOC_Os11g37640 & $1.80 \mathrm{e}+308$ & 0.0003495 & 0.0273744 & ADP-ribosylation factor, putative, expressed \\
\hline
\end{tabular}

Table 9. Differentially expressed genes (DEGs) related to signaling: Sample 3 vs Sample 1.

\begin{tabular}{lcccl}
\hline Gene_ID & $\log 2(\mathrm{FC})$ & P value & Q value & Description \\
\hline LOC_Os03g20700 & -1.38 & 0.0001318 & 0.0145273 & Protein magnesium-chelatase \\
LOC_Os11g37640 & $-1.80 \mathrm{e}+308$ & 0.0003495 & 0.0273744 & ADP-ribosylation factor \\
LOC_Os04g23890 & 1.43 & $4.50 \mathrm{E}-05$ & 0.0066587 & AGC_PVPK_like_kin82y.10-ACG kinases \\
LOC_Os02g06570 & $-1.80 \mathrm{e}+308$ & $2.87 \mathrm{E}-05$ & 0.0049155 & CAMK_KIN1/SNF1/Nim1_like.13-CAMK \\
LOC_Os11g34460 & 1.51 & 0.0002388 & 0.0213539 & OsFBO10-F-box and other domain-containing protein \\
LOC_Os08g08140 & $1.80 \mathrm{e}+308$ & 0.0006526 & 0.0415741 & Receptor-like kinase \\
LOC_Os12g04500 & -1.80 & 0.0003453 & 0.0271487 & Response regulator receiver domain-containing protein \\
LOC_Os01g16030 & -1.31 & 0.0001682 & 0.0170527 & ADP-ribosylation factor \\
LOC_Os09g25100 & 1.51 & 0.000161 & 0.0166126 & CAMK_KIN1/SNF1/Nim1_like.35-CAMK \\
LOC_Os01g01010 & $1.80 \mathrm{e}+308$ & 0.0007959 & 0.0467159 & TBC domain-containing protein \\
LOC_Os01g01010 & -2.73 & 0.0001098 & 0.0126794 & TBC domain-containing protein \\
\hline
\end{tabular}

\section{Low-energy ion-beam implantation re-activated transcription of some transpos- able elements (TEs)}

TEs are major components of most eukaryotic genomes and are particularly abundant in plants, representing $35 \%$ of the rice genome. TEs are critical in genome and gene evolution, often creating mutations and altering the cell's genome size (O'Donnell and Burns, 2010). TEs change selective gene expression because of non-coding RNA transcription from transposon promoters, which contribute to the epigenetic regulation of neighboring genes through mechanisms such as RNA interference, which can affect the expression of nearby genes (Zaratiegui et al., 2007). Thus, TE transcription was severely repressed in plants and was only activated under specific circumstances such as pathogenic infection, physical injury, or unique abiotic stresses (Qian et al., 2010). We reported that 14 DEGs in Sample 2 and 24 DEGs in Sample 3 are related to transposons or retrotransposons. In addition, seven DEGs related to TEs were differentially expressed both in Sample 2 and 3 compared to the control sample. Of these seven TE-related DEGs, five had induced expression in both Samples 2 and 3, and two were upregulated in both Samples 2 and 3 compared to the control sample (Table 10). Sequencing revealed that low-energy $\mathrm{N}^{+}$-beam implantation can re-activate the transcription of functional 
proteins related to TEs in rice, increasing the translocation potential of TEs in plant and contributing to plant genomic diversity and mutations.

\begin{tabular}{|c|c|c|c|}
\hline \multirow[t]{2}{*}{ Gene_ID } & \multicolumn{2}{|c|}{$\log 2(\mathrm{FC})$} & \multirow[t]{2}{*}{ Description } \\
\hline & Sample 2 vs Sample 1 & Sample 3 vs Sample 1 & \\
\hline LOC_Os11g43800 & $1.80 \mathrm{e}+308$ & $1.80 \mathrm{e}+308$ & Retrotransposon, Ty3-gypsy subclass \\
\hline LOC_Os10g37160 & 2.39 & 2.26192 & Transposon protein, putative, unclassified \\
\hline LOC_Os05g03120 & $1.80 \mathrm{e}+308$ & $1.80 \mathrm{e}+308$ & Protein retrotransposon protein, putative, unclassified \\
\hline $\mathrm{LOC}^{-} \mathrm{Os} 04 \mathrm{~g} 53660$ & $1.80 \mathrm{e}+308$ & $1.80 \mathrm{e}+308$ & Transposon protein, putative \\
\hline LOC_Os04g49780 & $1.80 \mathrm{e}+308$ & $1.80 \mathrm{e}+308$ & Transposon protein, CACTA, En/Spm sub-class \\
\hline LOC_Os03g36130 & 2.96 & 3.49934 & Retrotransposon, putative, centromere-specific \\
\hline LOC_Os02g30530 & $1.80 \mathrm{e}+308$ & $1.80 \mathrm{e}+308$ & Transposon protein, transposase, MuDR \\
\hline
\end{tabular}

\section{ACKNOWLEDGMENTS}

Research supported by the Key Technology Projects of Henan Province (Grant \#10210110108), the Program-Funded Project for Young Teachers in Universities of Henan Province (Grant \#2010ggjs-168), and the National Natural Science Fund of China (Grant \#30800204 and U1204307).

\section{Conflicts of interest}

All authors declare no conflict of interests.

\section{REFERENCES}

Benner S (2008). Biology from the bottom up. Nature 452: 692-694.

Chen H, Li F, Yuan H, Xiao X, et al. (2010). Abscopal signals mediated bio-effects in low-energy ion irradiated Medicago truncatula seeds. J. Radiat. Res. 51: 651-656.

Chen K and Rajewsky N (2007). The evolution of gene regulation by transcription factors and microRNAs. Nat. Rev. Genet. 8: 93-103.

Du Z, Zhou X, Ling Y, Zhang Z, et al. (2010). agriGO: a GO analysis toolkit for the agricultural community. Nucleic. Acids. Res. 38: W64-W70.

Feng H, Yu Z and Chu PK (2006). Ion implantation of organisms. Mater. Sci. Eng. Rep. 54: 49-120.

Feng H, Liu X, Yuan H, Kong M, et al. (2007). Utilizing low-energy ion beams to study living organisms. Surf. Coat. Tech. 201: 8034-8038.

Liu XD and Shen YG (2004). NaCl-induced phosphorylation of light harvesting chlorophyll a/b proteins in thylakoid membranes from the halotolerant green alga, Dunaliella salina. FEBS Lett. 569: 337-340.

Maekawa M, Hase Y, Shikazono N and Tanaka A (2003). Induction of somatic instability in stable yellow leaf mutant of rice by ion beam irradiation. Nucl. Instrum. Meth. B 206: 579-585.

Manning G, Whyte DB, Martinez R, Hunter T, et al. (2002). The protein kinase complement of the human genome. Science 298: 1912-1934.

Mortazavi A, Williams BA, McCue K, Schaeffer L, et al. (2008). Mapping and quantifying mammalian transcriptomes by RNA-Seq. Nat. Methods 5: 621-628.

Nemhauser JL, Feldman LJ and Zambryski PC (2000). Auxin and ETTIN in Arabidopsis gynoecium morphogenesis. Development 127: 3877-3888.

O'Donnell KA and Burns KH (2010). Mobilizing diversity: transposable element insertions in genetic variation and disease. Mob. DNA 1:21.

Qian Y, Cheng X, Liu Y, Jiang H, et al. (2010). Reactivation of a silenced minimal Mutator transposable element system following low-energy nitrogen ion implantation in maize. Plant. Cell. Rep. 29: 1365-1376. 
Robinson MD, McCarthy DJ and Smyth GK (2010). edgeR: a Bioconductor package for differential expression analysis of digital gene expression data. Bioinformatics 26: 139-140.

Scheeff ED and Bourne PE (2005). Structural evolution of the protein kinase-like superfamily. PLoS Comput. Biol. 1: e49. Shikazono N, Tanaka A, Watanabe H and Tano S (2001). Rearrangements of the DNA in carbon ion-induced mutants of Arabidopsis thaliana. Genetics 157: 379-387.

Tanaka A, Shikazono N and Hase Y (2010). Studies on biological effects of ion beams on lethality, molecular nature of mutation, mutation rate, and spectrum of mutation phenotype for mutation breeding in higher plants. J. Radiat. Res. 51: 223-233.

Teramoto H, Ono T and Minagawa J (2001). Identification of Lhcb gene family encoding the light-harvesting chlorophylla/b proteins of photosystem II in Chlamydomonas reinhardtii. Plant Cell. Physiol. 42: 849-856.

Wang Y, Chen KP and Yao Q (2008). Progress of studies on bHLH transcription factor families. Yi. Chuan 30: 821-830.

Wang Z, Gerstein M and Snyder M (2009). RNA-Seq: a revolutionary tool for transcriptomics. Nat. Rev. Genet. 10: 57-63.

Xia Y, Ning Z, Bai G, Li R, et al. (2012). Allelic variations of a light harvesting chlorophyll a/b-binding protein gene (Lhcb1) associated with agronomic traits in barley. PLoS One 7: e37573.

Ya H, Wang W, Chen W, Qin G, et al. (2010). Retrotransposon Tto1 in tobacco was activated by the implantation of lowenergy N+ ion beam. Life Sci. J. 7: 147.

Ya H, Chen Q, Wang W, Chen W, et al. (2012). Gene expression profiles in promoted-growth rice seedlings that germinated from the seeds implanted by low-energy $\mathrm{N}+$ beam. J. Radiat. Res. 53: 558-569.

Yamaguchi H, Nagatomi S, Morishita T and Degi K (2003). Mutation induced with ion beam irradiation in rose. Nucl. Instrum. Meth. B 206: 561-564.

Yamaguchi H, Shimizu A, Hase Y, Tanaka A, et al. (2010). Effects of ion beam irradiation on mutation induction and nuclear DNA content in chrysanthemum. Breed. Sci. 60: 398-404.

Zaratiegui M, Irvine DV and Martienssen RA (2007). Noncoding RNAs and gene silencing. Cell 128: 763-776. 